\title{
BMJ Open Changes in the quality of care of colorectal cancer in Estonia: a population-based high-resolution study
}

\author{
Heigo Reima (10 ,1,2 Jaan Soplepmann, ${ }^{1,2}$ Anneli Elme, ${ }^{3}$ Mari Lõhmus, ${ }^{3}$ Rena Tiigi, ${ }^{3}$ \\ Denis Uksov, ${ }^{4}$ Kaire Innos (1) ${ }^{5}$
}

To cite: Reima H,

Soplepmann J, Elme A, et al.

Changes in the quality of care of colorectal cancer in Estonia: a population-based highresolution study. BMJ Open 2020;10:e035556. doi:10.1136/ bmjopen-2019-035556

- Prepublication history and additional material for this paper are available online. To view these files, please visit the journal online (http://dx.doi. org/10.1136/bmjopen-2019035556).

Received 06 November 2019 Revised 31 August 2020 Accepted 10 September 2020

Check for updates

(c) Author(s) (or their employer(s)) 2020. Re-use permitted under CC BY-NC. No commercial re-use. See rights and permissions. Published by BMJ.

${ }^{1}$ Department of Surgical Oncology, Tartu University Hospital, Tartu, Estonia ${ }^{2}$ Institute of Clinical Medicine, University of Tartu, Tartu, Estonia ${ }^{3}$ Oncology and Haematology Clinic, North Estonia Medical Centre, Tallinn, Estonia

${ }^{4}$ Surgery-Anaesthesiology Clinic, South-Estonian Hospital, Võru,

Estonia

${ }^{5}$ Department of Epidemiology and Biostatistics, Tervise Arengu Instituut, Tallinn, Estonia

Correspondence to

Dr Heigo Reima;

heigo.reima@kliinikum.ee

\section{ABSTRACT}

Objectives Large disparities in colorectal cancer (CRC) management and survival have been observed across Europe. Despite recent increases, the survival deficit of Estonian patients with CRC persists, particularly for rectal cancer. The aim of this study was to examine diagnostic, staging and treatment patterns of CRC in Estonia, comparing clinical data from 1997 and 2011.

Design Nationwide population-based retrospective study. Setting Estonia.

Participants All incident cases of colon and rectal cancer diagnosed in 1997 and 2011 identified from the Estonian Cancer Registry. Clinical data gathered from medical records.

Outcome measures Differences in diagnostic, staging and treatment patterns; 5-year relative survival ratios. Results The number of colon cancer cases was 337 in 1997 and 498 in 2011; for rectal cancer, the respective numbers were 209 and 349. From 1997 to 2011, large increases were seen in the use of colonoscopy and lung and liver imaging. Radical resection rate increased from $48 \%$ to $59 \%$, but emergency surgeries showed a rise from $18 \%$ to $26 \%$ in colon and from $7 \%$ to $14 \%$ in rectal cancer. The proportion of radically operated patients with $\geq 12$ lymph nodes examined pathologically increased from $2 \%$ to $58 \%$ in colon cancer and from $2 \%$ to $50 \%$ in rectal cancer. The use of neoadjuvant radiotherapy increased from $6 \%$ to $39 \%$ among stage II and from $20 \%$ to $50 \%$ among patients with stage III rectal cancer. The use of adjuvant chemotherapy in stage III colon cancer increased from $42 \%$ to $63 \%$. The 5 -year RSR increased from $50 \%$ to $58 \%$ in colon cancer and from $37 \%$ to $64 \%$ in patients with rectal cancer.

Conclusions Major improvements were seen in the diagnostics, staging and treatment of CRC in Estonia contributing to better outcomes. Increase in emergency surgeries highlights possible shortcomings in timely diagnosis and treatment.

\section{INTRODUCTION}

Colorectal cancer (CRC) is the secondleading cause of cancer-related deaths in Europe. ${ }^{1}$ The incidence of CRC has increased, while survival rates have steadily improved in Europe as well as in Estonia. ${ }^{23}$ Diagnostic approaches, multimodality treatment and surgical techniques of CRC have

\section{Strengths and limitations of this study}

A population-based sample drawn from the nationwide cancer registry.

- Uniform data gathering from patient records by specialised doctors according to EUROCARE HighResolution study protocols and ability to directly compare two incidence years.

- Retrospective nature of data collection may have led to some bias due to inaccurate or missing data in medical records.

gone through significant changes during the past decades. ${ }^{4}$

In the EUROCARE-3 study (1990-1994), large variations in CRC survival were observed across Europe, with particularly poor rates for Eastern European countries, including Estonia. ${ }^{5}$ The 5-year relative survival of Estonian patients was below $40 \%$ for colon and around $30 \%$ for rectal cancer, while the European average for both sites was close to $50 \% .^{5}$

These observed variations between different countries prompted the initiation of European High-Resolution studies to examine the patterns of cancer care. In the late 1990s, the proportion of radically operated patients with CRC varied from $44 \%$ to $86 \%$ among the participating 12 registries with the estimate for Estonia (56\%) being one of the lowest. ${ }^{6}$ Shortcomings in diagnostics were evident as the proportion of cases resected radically who had $\geq 12$ lymph nodes examined was below $2 \%$ in Estonia while the European average was 29\%. ${ }^{6}$ A large variation $(24 \%-73 \%)$ was also seen in the use of adjuvant chemotherapy in stage III colon cancer. ${ }^{6}$

A more detailed analysis of the 1997 Estonian CRC cohort showed extremely low quality of pathological examination, inadequate staging and insufficient use of neoadjuvant and adjuvant therapies. ${ }^{7}$

A recent analysis of Estonian Cancer Registry data demonstrated that both overall 
and stage-specific relative survival of CRC patients have improved significantly and the survival gap between Estonia and the leading countries in Europe has narrowed. ${ }^{3}$ From 1995-1999 to 2010-2014, the 5-year relative survival increased the most for stage III rectal cancer (from $34 \%$ to $70 \%$ ), while manyfold increase was also seen for stage IV CRC. ${ }^{3}$ It was hypothesised that more accurate diagnostics and improved treatment have contributed to these changes.

This study was undertaken to examine the changes in diagnostic, staging and treatment patterns in Estonia since late 1990s, comparing clinical data from 1997 and 2011.

\section{MATERIAL AND METHODS}

Both study cohorts (1997 and 2011) were initially formed for European Cancer Registry (EUROCARE) European High-Resolution Studies, in order to compare cancer management practices across Europe. The study cohorts were identified from the Estonian Cancer Registry, which is a population-based registry covering the whole country (population 1.3 million in 2011) and has data since 1968. All incident cases of cancer of colon ((International Classification of Diseases (ICD-10) C18) and rectum (ICD-10 C19-20), diagnosed in adults (age $\geq 15$ years) in 1997 and 2011 were included. Death certificate only and autopsy cases ( $\mathrm{n}=16$ in 1997, $\mathrm{n}=13$ in 2011) were excluded.

Data on diagnostic procedures, staging and treatment were gathered retrospectively from the patients' medical records by oncologists and colorectal surgeons, according to the study protocols of EUROCARE High-Resolution Studies. ${ }^{68}$

Subsites were categorised into right-sided colon cancer, including appendix, caecum, ascending colon, hepatic flexure and transverse colon (ICD-10 C18.0-18.4); leftsided colon cancer, including splenic flexure, descending and sigmoid colon (C18.5-18.7); other or overlapping colon (C18.8-18.9); rectosigmoid junction (C19) and rectum (C20).

The stage (I-IV) was classified according to the tumour, nodes, metastases (TNM) Classification V.7 based on the algorithm shown in online supplemental table 1. Pathological TNM was used for staging primarily operated cases. If pathological TNM was missing or patients received neoadjuvant treatment, clinical TNM stage was used for the 2011 cohort. The 1997 study protocol did not include data collection on clinical $\mathrm{T}$ and $\mathrm{N}$ stage.

Surgical treatment was categorised as no surgery, radical surgery, not radical surgery, unknown as shown in online supplemental table 1 .

The equality of proportions in the 1997 and 2011 cohorts was tested with two-sample tests of proportions using large-sample statistics in STATA V.14 (StataCorp). Case counts below five are presented as ' $<5$ '.

Follow-up for vital status was conducted by the Estonian Cancer Registry at the Estonian Population Registry using unique personal identification numbers. In case of death or emigration, the respective dates were obtained. Survival time was accumulated from the date of diagnosis until the date of death; for patients who remained alive, survival time was censored at the time of emigration or at the end of follow-up, 31 December 2016.

Relative survival, which captures both direct and indirect mortality due to cancer, was used for survival analysis. ${ }^{9}$ Relative survival ratio (RSR) was calculated as the ratio of observed survival and expected survival of the underlying general population. The latter was calculated according to Ederer II method, ${ }^{10}$ based on national life tables of general-population survival probabilities, stratified by age (in 1-year increments from 0 to 99), sex and calendar year (in 1-year intervals). Patients who were diagnosed and died on the same day were included with 1 day of survival time. RSRs were calculated using the strs command in STATA V.14 (StataCorp) ${ }^{11}$ using actuarial methods and are presented with 95\% CI. Relative survival estimates were age standardised using direct standardisation with International Cancer Survival Standards. ${ }^{12}$

The study protocol was approved by Tallinn Medical Research Ethics Committee (Decision No. 556, 13 February 2014).

We used the Strengthening the Reporting of Observational Studies in Epidemiology cohort checklist when writing our report. ${ }^{13}$

\section{RESULTS}

The number of newly diagnosed CRC cases in Estonia was 546 in 1997 and 847 in 2011 (table 1). Age distribution shifted towards the elderly as the proportion of patients diagnosed at age $\geq 75$ increased for both colon and rectal cancer. In colon cancer, there was a shift towards rightsided colon cancers. The proportion of stage III disease increased the most among colon and rectal cancer patients. The proportion of cases diagnosed at stage IV did not change.

From 1997 to 2011, the use of colonoscopy increased and the use of double contrast barium enema (DCBE) decreased in colon cancer patients (table 2). Among elective colon cancer patients, the use of colonoscopy was $82 \%$ in 1997 and $91 \%$ in 2011 ( $\mathrm{p}=0.0046$ ). Pulmonary and liver imaging for detecting distant metastases was more frequently used in 2011 both in colon and rectal cancer patients. Multidisciplinary team meetings (MDTM) were not practiced routinely in 1997 in Estonia. In 2011, treatment plan was determined in MDTM for the majority of CRC patients. Among patients who underwent elective surgery, the proportion of patients discussed in MDTM was $65 \%$ for colon and $86 \%$ for rectal cancer. The proportion of patients who had $\geq 12$ lymph nodes examined pathologically increased from $2 \%$ to $58 \%$ in colon and from $2 \%$ to $50 \%$ in patients with rectal cancer.

The proportion of patients receiving radical surgery increased both in colon and rectal cancer patients (table 3). At the same time, there was an increase in the proportion of emergency surgery among surgically 
Table 1 Characteristics of the 1997 and 2011 colorectal cancer cohorts in Estonia

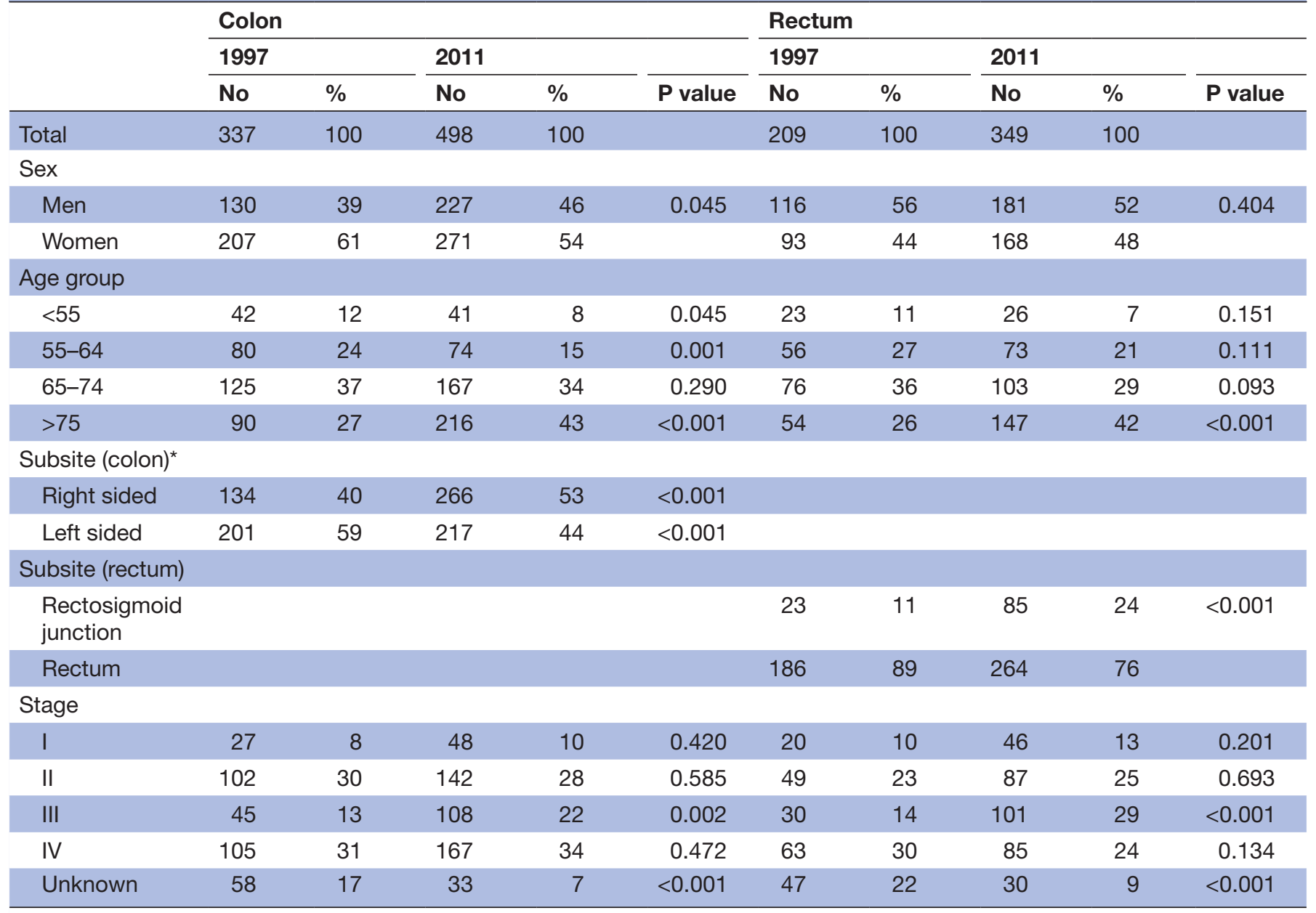

*Overlapping and unspecified subsites not presented in the table due to small numbers.

treated patients. In 2011, more temporary colostomies/ ileostomies were used and less permanent colostomies were done in rectal cancer. Postoperative 30-day mortality rate decreased among patients with colon cancer.

In 1997, all colorectal resections were done by laparotomy, whereas in 2011, the proportion of laparoscopic surgery among elective procedures was $12 \%$ in colon and $8 \%$ in rectal cancer. In $2011,5 \%$ of rectal cancer patients had their tumour removed by local excision. Radical surgery with the removal of all metastases was performed in $14 \%(23 / 167)$ of patients with stage IV colon and $13 \%$ $(11 / 85)$ of stage IV rectal cancer in 2011.

The use of neoadjuvant radiotherapy or chemotherapy increased among patients with stage II-IV rectal cancer (table 4). The use of adjuvant chemotherapy increased among patients with stage II-III colon cancer and patients with stage III rectal cancer.

From 1997 to 2011, the 5-year age-standardised RSR increased from $50 \%(95 \%$ CI $43 \%$ to $57 \%)$ to $58 \%(95 \%$ CI $52 \%$ to $63 \%)$ in colon cancer and from $37 \%$ (95\% CI $29 \%$ to $45 \%)$ to $64 \%(95 \%$ CI $57 \%$ to $69 \%$ ) in patients with rectal cancers (figure 1). Among all radically operated patients in 2011, the 5-year RSR was $81 \%$ (95\% CI
$74 \%$ to $87 \%$ ) for colon and $88 \%$ (79\% to $95 \%)$ for rectal cancer; the respective RSRs in stage I-III radically operated patients were $84 \%(95 \%$ CI $74 \%$ to $91 \%)$ and $91 \%$ (95\% CI $82 \%$ to $98 \%$ ).

\section{DISCUSSION}

The number of CRC cases diagnosed annually has markedly increased in Estonia over 14 years (in comparison of 1997 and 2011). Major improvements were seen in diagnostic, staging and treatment process, which have likely contributed to the significantly improved survival outcomes observed in Estonia. ${ }^{3}$

The main strength of the study was the populationbased sample drawn from the nationwide cancer registry. Reporting to the registry in Estonia is mandatory by law. Another strength was uniform data gathering from patient records by specialised doctors according to EUROCARE High-Resolution study protocols and ability to directly compare two incidence years. The weakness of the study was the retrospective nature of data collection. Inaccurate or missing data in medical records could have led to some bias. Owing to some differences between 1997 and 
Table 2 Diagnostics in the 1997 and 2011 colorectal cancer cohorts in Estonia

\begin{tabular}{|c|c|c|c|c|c|c|c|c|c|c|}
\hline & \multicolumn{5}{|c|}{ Colon } & \multicolumn{5}{|c|}{ Rectum } \\
\hline & \multicolumn{2}{|c|}{1997} & \multicolumn{2}{|c|}{2011} & \multirow[b]{2}{*}{$P$ value } & \multicolumn{2}{|c|}{1997} & \multicolumn{2}{|c|}{2011} & \multirow[b]{2}{*}{$P$ value } \\
\hline & No & $\%$ & No & $\%$ & & No & $\%$ & No & $\%$ & \\
\hline No of cases & 337 & 100 & 498 & 100 & & 209 & 100 & 349 & 100 & \\
\hline \multicolumn{11}{|l|}{ Intestinal examinations } \\
\hline Lung imaging & 194 & 58 & 436 & 88 & $<0.001$ & 149 & 71 & 312 & 89 & $<0.001$ \\
\hline Thorax CT & - & & 381 & 77 & & - & & 291 & 83 & \\
\hline Liver imaging & 230 & 68 & 472 & 95 & $<0.001$ & 165 & 79 & 335 & 96 & $<0.001$ \\
\hline Abdominal CT & - & & 427 & 86 & & - & & 311 & 89 & \\
\hline \multicolumn{11}{|c|}{ No of lymph nodes examined among radically operated } \\
\hline$>12$ & $<5$ & 2 & 169 & 58 & $<0.001$ & $<5$ & 2 & 105 & 50 & $<0.001$ \\
\hline $9-11$ & $<5$ & 1 & 40 & 14 & $<0.001$ & $<5$ & 1 & 25 & 12 & 0.001 \\
\hline$<9$ & 74 & 45 & 66 & 23 & $<0.001$ & 54 & 55 & 64 & 31 & $<0.001$ \\
\hline Unknown & 86 & 52 & 14 & 5 & $<0.001$ & 42 & 42 & 15 & 7 & $<0.001$ \\
\hline
\end{tabular}

2011 study protocols, we were not able to compare all the parameters directly. According to the 1997 study protocol, data on clinical $\mathrm{T}$ and $\mathrm{N}$ status were not collected. Thus, the proportion of unknown stage was considerably higher for the 1997 cohort. Small sample size did not allow us to obtain reliable results for stage-specific survival.

Estonian population is 1.3 million. The majority of patients with CRC are treated in two cancer centres offering full range of cancer treatment modalities. Cancer care in Estonia is fully covered by public insurance. CRC population-based screening programme in Estonia was initiated in 2016 and did not affect our findings. Faecal occult blood test is carried out at age 60 and repeated every 2 years, the process is coordinated by family physicians. This is followed by colonoscopy in case of a positive test.

As shown previously, CRC incidence has increased significantly in Estonia. ${ }^{3}$ There was a shift in the age distribution towards older age groups, as the proportion of patients diagnosed at the age of $\geq 75$ years increased significantly, probably associated with population ageing. There was also an increase in right-sided colon cancer incidence and decrease in left-sided colon cancer incidence. This phenomenon has been described elsewhere. ${ }^{1415}$ The reasons for the increase in right-sided colon cancer are not clear, but CRC subsites may have different aetiology in terms of inherited and environmental factors. ${ }^{16}$ We also found a shift in the stage distribution as the proportion of stage III patients increased both in colon and rectal cancer cohorts. This can be explained by significantly better lymph node examination and more accurate clinical staging with the use of MRI for patients with rectal cancer who received neoadjuvant therapy.

Colonoscopy with biopsy is the main procedure for CRC diagnosis according to European Society for Medical Oncology (ESMO) clinical practice guidelines. ${ }^{1718}$ The lower rate of colonoscopy and frequent use of DCBE in 1997 could probably be explained by lower availability of endoscopy and DCBE being an accessible alternative. The availability of endoscopy has improved in Estonia and there are virtually no indications left for DCBE. In emergency cases with bowel obstruction, diagnosis is usually based on emergency department CT findings and these patients receive surgery without prior endoscopy. In our data, around $20 \%$ of surgically treated patients were operated in emergency situation, this group includes mostly the patients whose diagnosis was not based on colonoscopy. Among patients who underwent elective colon cancer surgery, colonoscopy use exceeded $90 \%$ in 2011. This result is similar to that shown for Switzerland. ${ }^{19}$

Pelvic MRI is the most accurate modality for rectal cancer locoregional clinical staging. ${ }^{18}$ Another locoregional staging tool alternative to MRI is transrectal endosonography, but this was not routinely used in Estonia in 2011. In 1997, MRI was not available in Estonia. In 2011, only $36 \%$ of rectal cancer patients underwent MRI, which was probably a consequence of still low availability. Accessibility of MRI has improved lately in Estonia and currently, the majority of rectal cancer MDTM discussions are based on MRI imaging. 


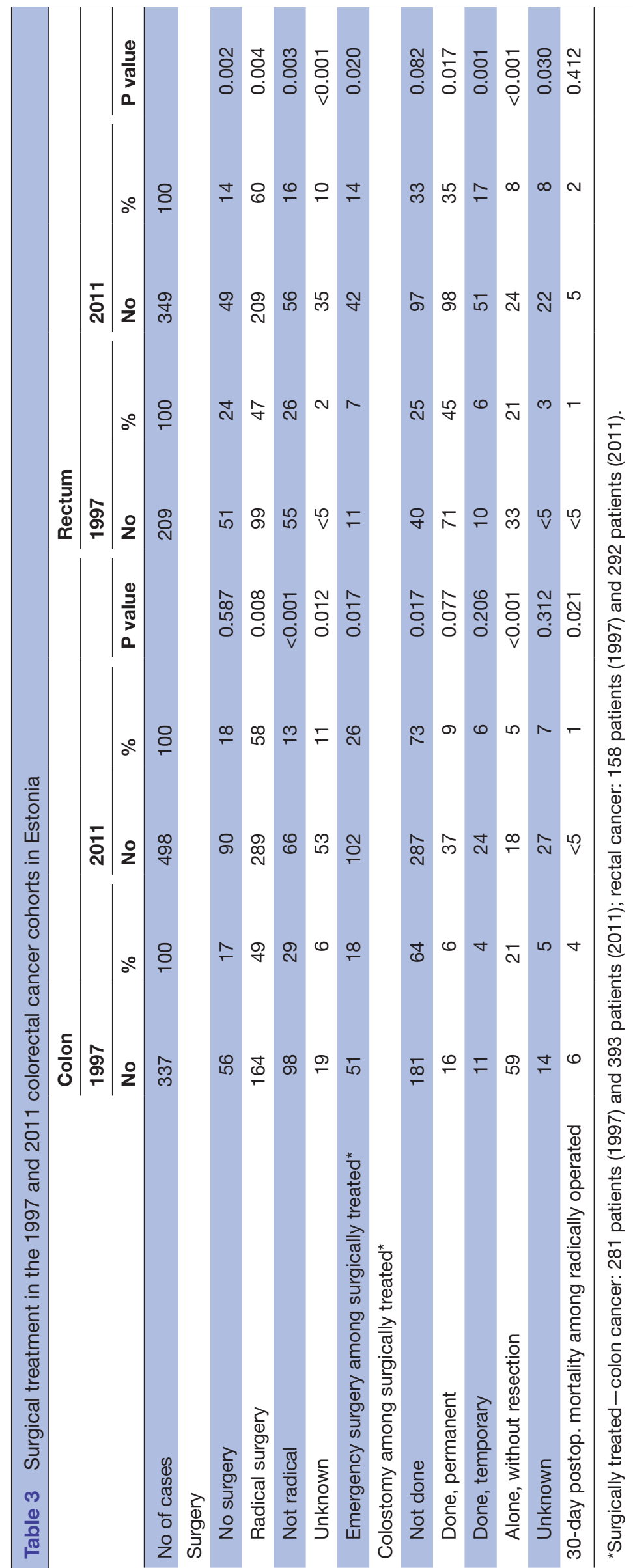

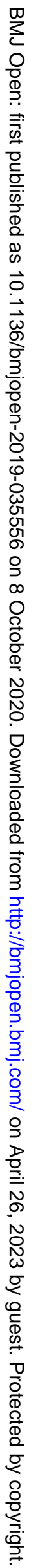


Table 4 Radiotherapy and chemotherapy by stage in the 1997 and 2011 colorectal cancer cohorts in Estonia

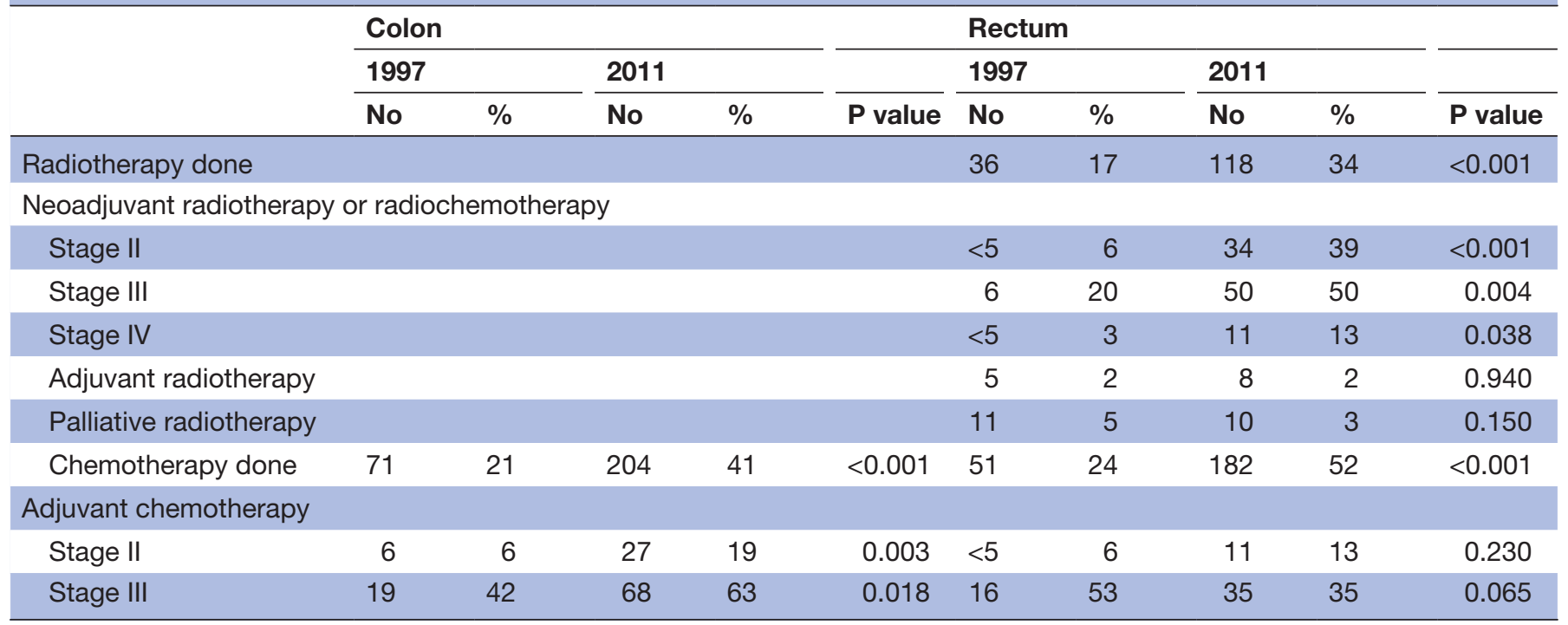

Thoracic and abdominal imaging are important for detecting distant metastases of CRC, which are mostly located in the liver, lungs and peritoneum. In our findings, the use of thoracic and liver imaging improved significantly. Contrast-enhanced CT is the preferred imaging modality. Alternatively, thoracic X-ray and abdominal ultrasound can be used, but they have lower sensitivity and specificity. ${ }^{20}$ The 1997 study protocol did not differentiate between imaging modalities. In 2011, the majority of cases with imaging information available underwent thoracic and abdominal CT.

According to ESMO clinical practice guidelines, rectal cancer treatment plan should be established during MDTM. ${ }^{18}$ This was not the routine practice in Estonia in 1997, but in 2011, $86 \%$ of elective patients with rectal cancer were discussed at MDTM. This indicator is comparable with data shown for Sweden in the same year (around 90\%). ${ }^{21}$ MDTM had limited impact on patient survival, but patients discussed were more likely to receive complete preoperative staging and neoadjuvant/adjuvant treatment. ${ }^{22}$ There are centres discussing all incident CRC cases. On the other hand, there are authors who suggest that discussing routine cases would
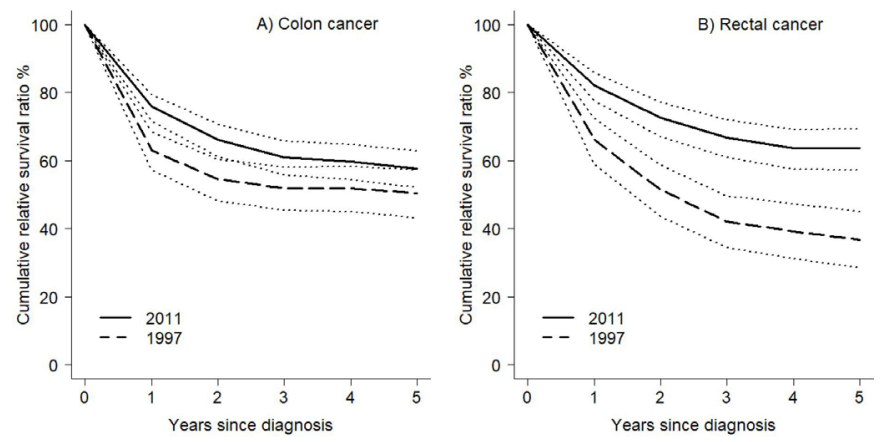

Figure 1 Cumulative age-standardised relative survival ratios of the 1997 and 2011 cancer cohorts in Estonia, (A) colon cancer; $(\mathrm{B})$ rectal cancer. not change the treatment plan and could be a waste of time and money. They advocate discussing only complex cases. $^{23} 24$ Emergency patients are operated without MDTM decision. MDTM is especially important in rectal cancer and oligometastatic CRC, where decision making is more complex. In Sweden, around $60 \%$ of patients with colon cancer receiving elective surgery were discussed in a preoperative MDTM in 2011, but the indicator has reached $93 \%$ by $2017 .^{25}$

Surgical resection is a crucial part of curative treatment for CRC. In 2011, a significantly higher proportion of patients were operated radically than in 1997. The largest increase in the proportion of radically operated patients was seen in age group $\geq 75$ years (from $30 \%$ to $53 \%, \mathrm{p}<0.001$; data not shown). Thirty-day postoperative mortality among radically operated patients with colon cancer decreased despite that there were significantly more elderly $(\geq 75)$ patients in the latter period. As expected, 30-day postoperative mortality was much higher after emergency surgery compared with elective surgery ( $10 \%$ vs $2 \%$, data not shown), which is consistent with previous reports. ${ }^{19}$ The reduction of postoperative CRC mortality has been described elsewhere. ${ }^{26}$ The reasons behind the decrease in surgery-related mortality can be associated with better perioperative patient management: patient optimisation, application of enhanced recovery protocol, using minimally invasive surgery, advances in complication prevention and management; as well as with advances in intensive care.

Rectal cancer surgery has also evolved towards function-sparing procedures. In our study, significantly more sphincter-preserving operations were done in rectal cancer patients in 2011 as the proportion of permanent colostomies decreased and the use of temporary ileostomy (which are mostly closed 2 months after index operation) increased. In a British analysis, abdominoperineal excision rate decreased from 29\% to $21 \%$ between 1996 
and $2004 .^{27}$ This was explained by clearer definition of the safe distal resection margin, evolution of surgical techniques (total mesorectal excision (TME) principles, application of stapling devices in low rectal anastomosis) and use of neoadjuvant radiotherapy. ${ }^{27}$ We found that in $5 \%$ of the rectal cancer cases, the tumour was removed by local excision in 2011. This less invasive approach is indicated in selected patients with $\mathrm{T} 1$ cancer. $^{28}$ With gradual expansion of CRC screening programme in Estonia, we expect to diagnose more $\mathrm{T} 1$ cancers who are suitable for local excision procedure.

In 1997, all CRC resections were done by open surgery. In 2011, the proportion of laparoscopic procedures was $12 \%$ in colon and $8 \%$ in patients with rectal cancer among elective surgeries, this is comparable to Swedish data at that time. ${ }^{21}{ }^{25}$ However, the use of laparoscopic surgery in CRC has further increased in Estonia as well as in Europe. ${ }^{25}$ An analysis of 2017 data showed that at Tartu University Hospital (the second-largest cancer centre in Estonia), $45 \%$ of CRC resections were done laparoscopically (data not published).

The 1997 study protocol did not include data collection on the resection of metastases in stage IV CRC. In 2011, our complete resection rate for metastatic disease was $14 \%$ in colon and $13 \%$ in rectal cancer. Complete (R0) removal of metastases offers the only chance of cure for patients with advanced CRC. In a systematic review, the median of 5-year survival estimates among patients who received complete metastasectomy was $38 \% .{ }^{29}$ Liver resection rates ranging from $10 \%$ to $30 \%$ were reported. The rate of curative surgery for metastatic CRC has improved with perioperative use of combination therapy regimens including biological therapy. ${ }^{30}$ A recent analysis showed that the 5-year RSR for stage IV CRC in Estonia was 13\%. ${ }^{3}$ This is comparable with respective estimate from Sweden $(15 \%){ }^{31}$

We observed an unexpected unfavourable trend as the proportion of emergency surgeries increased significantly. In 2011, every fourth colon cancer surgery was performed after emergency admission, a similar estimate was reported for Sweden. ${ }^{25}$ The main reason for both the persistently high proportion of stage IV patients and the observed increase in emergency surgery patients (most of them with bowel obstruction) is probably delay in diagnosis. This could be caused by lack of screening (population-based CRC screening was initiated in Estonia in 2016) and low awareness about CRC symptoms among general public as well as primary care doctors resulting in delayed presentation and referral. Another problem could be the delay of treatment due to long waiting period for the staging procedures and treatment. ${ }^{323}$

In locoregionally advanced rectal cancer, preoperative radiotherapy is associated with lower risk of local recurrence and better outcomes. ${ }^{34}$ In 1997 , neoadjuvant radiotherapy was not in routine clinical practice in Estonia and only $6 \%$ of stage II and $20 \%$ of stage III patients with rectal cancer received this treatment. The receipt of radiotherapy has been affected by limited availability as the relative number of linear accelerators in Estonia has been one of the lowest in Europe according to the ESTRO-HERO survey. ${ }^{35}$ This situation had improved by 2011 when $39 \%$ of stage II and $50 \%$ of stage III patients with rectal cancer received neoadjuvant radiotherapy, that accounts for half of all radically operated patients. In a recent Danish CRC registry study, the use of neoadjuvant radiotherapy was 29\% among radically operated patients with rectal cancer. ${ }^{36}$ In Norway, the use of neoadjuvant chemoradiotherapy was $39 \%$ among stage I-III radically operated patients with rectal cancer. ${ }^{37}$

Pathological examination of regional lymph nodes is crucial for accurate staging. International consensus standard suggests examination of at least 12 lymph nodes from a resected CRC specimen. ${ }^{38}$ In 1997, this standard was not adopted in Estonia and only $2 \%$ of radically operated patients had $\geq 12$ lymph nodes examined. Therefore, a significant proportion of patients were probably understaged. By 2011, the quality of pathology had significantly improved as 58\% patients with colon cancer and 50\% patients with rectal cancer had $\geq 12$ lymph nodes examined. Nevertheless, these results remain considerably worse than those shown for Norway ${ }^{39}$ or Switzerland ${ }^{19}$ for the same period. However, according to a trial including 266 patients conducted recently at Tartu University Hospital, $\geq 12$ lymph nodes were examined in $69 \%$ of conventionally investigated CRC specimens and $86 \%$ of methylene blue stained specimens, suggesting that the routine practice has further improved in Estonia. ${ }^{40}$

Colon cancer prognosis can be significantly improved by postoperative chemotherapy in stage III patients. ${ }^{41} \mathrm{In}$ our study, only $42 \%$ of patients with stage III colon cancer received adjuvant chemotherapy in 1997; in 2011, the respective percentage was $63 \%$. Among radically resected stage III patients, this proportion was 69\% (data not shown), while it was 59\% in Swsitzerland in 2011-2019. In comparison of 6 European countries, the proportion of resected stage III colon cancer receiving chemotherapy in Estonia was not inferior to Western European countries in 2011. ${ }^{42}$ The use of chemotherapy also increased in neoadjuvant and palliative settings.

Aforementioned advances in diagnostics, staging, multidisciplinary management and better surgical care are the most likely explanation for better long-term results as the 5-year RSR increased from $50 \%$ to $58 \%$ in colon cancer and from $37 \%$ to $64 \%$ in rectal cancer. These survival increases are in line with previously reported estimates. ${ }^{3}$ There was no definitive change towards earlier diagnosis. Due to small sample size in 1-year cohorts, we were not able to obtain reliable survival estimates by stage. However, a previous publication reported a large increase in stage-specific survival in Estonia: comparing two periods (1995-1999 and 2010-2014), a significant survival gain was seen for rectal cancer in stage II (from $58 \%$ to $75 \%$ ), stage III (from $34 \%$ to $70 \%$ ) and stage IV (from $1 \%$ to $12 \%$ ). For colon cancer, the largest survival gain was seen in stage IV (from 6\% to 15\%). ${ }^{3}$ These increases in survival are consistent with the improvements 
in staging and treatment patterns demonstrated in our study.

\section{CONCLUSIONS}

The findings of this study demonstrate the benefit of participating in EUROCARE studies that have revealed shortcomings in the quality of care in Estonia compared with most Northern and Western European countries and strengthened the motivation to take steps towards improvement. Major advances from 1997 to 2011 were seen in the diagnostic, staging and treatment patterns of CRC in Estonia: increase in the use of colonoscopy and imaging for distant metastases, introduction of MRI imaging for rectal cancer, higher rate of radical surgery with lower procedure-related mortality, substantial improvement in the quality of pathological examination and the adoption of multimodality treatment approach. These advances are likely to have contributed to improved survival shown for Estonia. Nevertheless, there is still room for improvement in all abovementioned areas. High proportion of patients diagnosed at a late stage and increasing rate of emergency surgeries remain an obstacle for achieving even better outcomes. These findings suggest the need to raise awareness about CRC symptoms among general public as well as among primary care doctors and to expand the quality-assured screening programme in Estonia. Continuous monitoring of performance and outcomes is necessary and further studies are warranted to examine diagnostic and treatment delays and centralisation of surgical treatment as possible areas of improvement.

Acknowledgements The authors thank EUROCARE High-Resolution Study team for planning and coordinating the studies, and Kristi Niinepuu and Sander Kütner for their help in collecting the data.

Contributors $\mathrm{HR}, \mathrm{Kl}$ and JS contributed to the study concept and design. HR, $\mathrm{KI}, \mathrm{JS}, \mathrm{AE}, \mathrm{ML}$, DU and RT participated in the acquisition of data. KI performed statistical analysis of the data. HR wrote the first and final draft of the paper. HR, $\mathrm{KI}, \mathrm{JS}, \mathrm{AE}, \mathrm{ML}$, DU and RT critically revised the manuscript for important intellectual content and approved the final version.

Funding The study was partly supported by Estonian Research Council (grant no. IUT5-1 and PRG722) and by the Ministry of Social Affairs of Estonia.

Competing interests None declared.

Patient consent for publication Not required.

Ethics approval The study protocol was approved by the Tallinn Medical Research Ethics Committee (approval no 556, 13.02.2014).

Provenance and peer review Not commissioned; externally peer reviewed.

Data availability statement Data are available on reasonable request.

Open access This is an open access article distributed in accordance with the Creative Commons Attribution Non Commercial (CC BY-NC 4.0) license, which permits others to distribute, remix, adapt, build upon this work non-commercially, and license their derivative works on different terms, provided the original work is properly cited, appropriate credit is given, any changes made indicated, and the use is non-commercial. See: http://creativecommons.org/licenses/by-nc/4.0/.

\section{ORCID iDs}

Heigo Reima http://orcid.org/0000-0001-6036-8008

Kaire Innos http://orcid.org/0000-0003-0120-2213
REFERENCES

1 Ferlay J, Colombet M, Soerjomataram I, et al. Cancer incidence and mortality patterns in Europe: estimates for 40 countries and 25 major cancers in 2018. Eur J Cancer 2018;103:356-87.

2 De Angelis R, Sant M, Coleman MP, et al. Cancer survival in Europe 1999-2007 by country and age: results of EUROCARE-5-a population-based study. Lancet Oncol 2014;15:23-34.

3 Innos K, Reima H, Baburin A, et al. Subsite- and stage-specific colorectal cancer trends in Estonia prior to implementation of screening. Cancer Epidemiol 2018;52:112-9.

4 Ahmed S, Johnson K, Ahmed O, et al. Advances in the management of colorectal cancer: from biology to treatment. Int $J$ Colorectal Dis 2014;29:1031-42.

5 Sant M, Aareleid T, Berrino F, et al. EUROCARE-3: survival of cancer patients diagnosed 1990-94-results and commentary. Ann Oncol 2003;14:v61-118.

6 Gatta G, Zigon G, Aareleid T, et al. Patterns of care for European colorectal cancer patients diagnosed 1996-1998: a EUROCARE high resolution study. Acta Oncol 2010;49:776-83.

7 Innos K, Soplepmann J, Suuroja T, et al. Survival for colon and rectal cancer in Estonia: role of staging and treatment. Acta Oncol 2012;51:521-7.

8 EPAAC. European high-resolution studies, 2012. Available: http:// www.hrstudies.eu

9 Dickman PW, Adami H-O. Interpreting trends in cancer patient survival. J Intern Med 2006;260:103-17.

10 Ederer $\mathrm{F}$, Heise $\mathrm{H}$. Instructions to IBM 650 programmers in processing survival computations. methodological note No. 10. Bethesda, MD: End Results Evaluation Section, National Cancer Institute, 1959.

11 Dickman PW, Coviello E. Estimating and modeling relative survival. Stata J 2015;15:186-215.

12 Corazziari I, Quinn M, Capocaccia R. Standard cancer patient population for age standardising survival ratios. Eur J Cancer 2004;40:2307-16.

13 von Elm E, Altman DG, Egger M, et al. The strengthening the reporting of observational studies in epidemiology (STROBE) statement: guidelines for reporting observational studies. Int $J$ Surg 2014;12:1495-9.

14 Cheng L, Eng C, Nieman LZ, et al. Trends in colorectal cancer incidence by anatomic site and disease stage in the United States from 1976 to 2005. Am J Clin Oncol 2011;34:573-80.

15 Papagiorgis PC, Oikonomakis I, Delaportas D, et al. Proximal shift of colorectal cancer. A persistent phenomenon with multiple causes, patterns and clinical implications. J Buon 2014;19:605-17.

16 Lee $\mathrm{GH}$, Malietzis G, Askari A, et al. Is right-sided colon cancer different to left-sided colorectal cancer? - a systematic review. Eur J Surg Oncol 2015;41:300-8.

17 Labianca R, Nordlinger B, Beretta GD, et al. Early colon cancer: ESMO clinical practice guidelines for diagnosis, treatment and follow-up. Ann Oncol 2013;24:vi64-72.

18 Glynne-Jones R, Wyrwicz L, Tiret E, et al. Rectal cancer: ESMO clinical practice guidelines for diagnosis, treatment and follow-up. Ann Oncol 2017;28:iv22-40.

19 Spitale A, Ortelli L, Mazzucchelli L, et al. Quality indicators of colorectal cancer care in southern Switzerland: results from a population-based study. Swiss Med Wkly 2017;147:w14530.

20 Balthazar EJ, Megibow AJ, Hulnick D, et al. Carcinoma of the colon: detection and preoperative staging by CT. AJR Am J Roentgenol 1988;150:301-6.

21 Rektalrapport. Rektalcancer2017 Nationell kvalitetsrapport för år 2017 från Svenska Kolorektalcancerregistret. Available: http://www. cancercentrum.se/globalassets/cancerdiagnoser/tjock-och-andtarmanal/kvalitetsregister/tjock-och-andtarm-fr.-2018/rektalrapport_2017. pdf2018

22 Pillay B, Wootten AC, Crowe $\mathrm{H}$, et al. The impact of multidisciplinary team meetings on patient assessment, management and outcomes in oncology settings: a systematic review of the literature. Cancer Treat Rev 2016;42:56-72.

23 Meagher AP. Colorectal cancer: are multidisciplinary team meetings a waste of time? ANZ J Surg 2013;83:101-3.

24 Ryan J, Faragher I. Not all patients need to be discussed in a colorectal cancer MDT meeting. Colorectal Dis 2014;16:520-6.

25 Kolonrapport. Koloncancer. Nationell kvalitetsrapport för år 2017 från Svenska Kolorektalcancerregistret. Available: http://www. cancercentrum.se/globalassets/cancerdiagnoser/tjock-och-andtarmanal/kvalitetsregister/tjock-och-andtarm-fr.-2018/kolonrapport_2017. pdf2018

26 Iversen LH, Ingeholm P, Gögenur I, et al. Major reduction in 30-day mortality after elective colorectal cancer surgery: a nationwide population-based study in Denmark 2001-2011. Ann Surg Oncol 2014;21:2267-73. 
27 Tilney HS, Heriot AG, Purkayastha S, et al. A national perspective on the decline of abdominoperineal resection for rectal cancer. Ann Surg 2008;247:77-84

28 Junginger T, Goenner U, Hitzler M, et al. Long-term oncologic outcome after transanal endoscopic microsurgery for rectal carcinoma. Dis Colon Rectum 2016;59:8-15.

29 Kanas GP, Taylor A, Primrose JN, et al. Survival after liver resection in metastatic colorectal cancer: review and meta-analysis of prognostic factors. Clin Epidemiol 2012;4:283-301.

30 Nordlinger B, Van Cutsem E, Rougier P, et al. Does chemotherapy prior to liver resection increase the potential for cure in patients with metastatic colorectal cancer? A report from the European colorectal metastases treatment group. Eur J Cancer 2007;43:2037-45.

31 Regionalt Cancercentrum Norr. Nationell Patientrapport 2016. Regionalt Cancercentrum. Norr, Norrlands universitetssjukhus, Umeå, 2017. https://www.cancercentrum.se/globalassets/ cancerdiagnoser/tjock--och-andtarm-anal/kvalitetsregister/rapporter2017/patientrapport2016.pdf

32 Courtney RJ, Paul CL, Sanson-Fisher RW, et al. Current state of medical-advice-seeking behaviour for symptoms of colorectal cancer: determinants of failure and delay in medical consultation. Colorectal Dis 2012;14:e222-9.

33 Domínguez-Ayala M, Díez-Vallejo J, Comas-Fuentes A. Missed opportunities in early diagnosis of symptomatic colorectal cancer. Rev Esp Enferm Dig 2012;104:343-9.

34 Kapiteijn E, Marijnen CA, Nagtegaal ID, et al. Preoperative radiotherapy combined with total mesorectal excision for resectable rectal cancer. N Engl J Med 2001;345:638-46.
35 Grau C, Defourny N, Malicki J, et al. Radiotherapy equipment and departments in the European countries: final results from the ESTROHERO survey. Radiother Oncol 2014;112:155-64.

36 Poulsen L, Yilmaz MK, Ljungmann K, et al. Local recurrence rate in a national Danish patient cohort after curative treatment for rectal cancer. Acta Oncol 2018:1-7.

37 Guren MG, Kørner H, Pfeffer F, et al. Nationwide improvement of rectal cancer treatment outcomes in Norway, 1993-2010. Acta Oncol 2015;54:1714-22.

38 Fielding LP, Arsenault PA, Chapuis $\mathrm{PH}$, et al. Clinicopathological staging for colorectal cancer: an international documentation system (IDS) and an international comprehensive anatomical terminology (ICAT). J Gastroenterol Hepatol 1991;6:325-44.

39 Årsrapport 2008-2014 Tykktarmskreft 1996-2014 Endetarmskreft. Available: https://www.kreftregisteret.no/globalassets/publikasjonerog-rapporter/arsrapporter/publisert-2015/aarsrapport_2014_tykk_ og_endetarmskreft.pdf2015

40 Reima H, Saar H, Innos K, et al. Methylene blue intra-arterial staining of resected colorectal cancer specimens improves accuracy of nodal staging: A randomized controlled trial. Eur J Surg Oncol 2016;42:1642-6.

41 Andre T, de Gramont A, Study Group of Clinical Research in Radiotherapies Oncology, Oncology Multidiciplinary Research Group. An overview of adjuvant systemic chemotherapy for colon cancer. Clin Colorectal Cancer 2004;4:S22-8.

42 Minicozzi P, Vicentini M, Innos K, et al. Comorbidities, timing of treatments, and chemotherapy use influence outcomes in stage III colon cancer: a population-based European study. Eur J Surg Oncol 2020;46:1151-9. 